EPJ Web of Conferences 53, 01001 (2013)

DOI: $10.1051 /$ epjconf/20135301001

(C) Owned by the authors, published by EDP Sciences, 2013

\title{
Ultra-high energy cosmic rays: Setting the stage
}

\author{
P. Sokolsky \\ Department of Physics and Astronomy, University of Utah, Salt Lake City, Utah, 84112
}

\begin{abstract}
The history of ultra-high energy cosmic ray physics is reviewed from the post-war era of arrays such as Volcano Ranch, Haverah Park and Akeno to the development of air-fluorescence and current hybrid arrays. The aim of this paper is to present the background information needed for a better understanding of the current issues in this field that are discussed in much greater depth in the rest of this conference.
\end{abstract}

\section{INTRODUCTION}

This meeting coincides with an important anniversary in cosmic ray research. One hundred years ago, Victor Hess took a hot-air balloon up to $15,000 \mathrm{ft}$ and demonstrated conclusively that there was a component of natural radiation whose intensity increased with height above the surface of the Earth (Fig. 1). This would later be named "Cosmic Ray" radiation. In his report on the results of several flights, Hess concluded: "The results of these observations seem best explained by the existence of radiation of great penetrating power entering the atmosphere from above." Victor Hess received the Nobel Prize for this discovery 24 years later. Though much has been learned, one hundred years later, there is still much about this radiation that is mysterious and unknown.

We can briefly characterize the important periods in the study of cosmic rays as [1]:

a. Pre-history: the discovery of cosmic radiation and the study of its penetrating power. This led to great debates between Millikan and Compton about the kinds of particles that composed it. The discovery of the latitude effect followed by the East-West effect conclusively proved that the majority of the cosmic ray particles were positively charged (protons and nuclei).

b. Ancient history: the discovery that high energy cosmic radiation produced extensive air showers and that much of the cosmic radiation on the surface was the result of secondary processes in the atmosphere. This was also the era when the presence of very high energy particles in the primary radiation was clearly demonstrated (by B. Rossi, P. Auger and others).

c. Middle Ages: the post-war development of large area surface detectors covering areas up to $10 \mathrm{~km}^{2}$ coming from the pioneering work of B. Rossi., J. Linsley and others.

d. Renaissance: the development of fully calorimetric techniques based on air-fluorescence (by the Fly's Eye group, based on earlier work of J. Keuffel, K. Greisen and others) and the extension of ground arrays to $100 \mathrm{~km}^{2}$ (AGASA).

e. Modern times: the refinement of air-fluorescence techniques (HiRes) and the construction of hybrid air-fluorescence/surface array detectors of unprecedented scale (Pierre Auger Observatory (PAO), Telescope Array (TA)).

\section{POST-WAR DEVELOPMENTS}

Bruno Rossi, building on the work of P. Auger and his own experiments demonstrating the existence of extensive airs showers (EAS), began work at MIT on the first surface array of scintillation detectors

This is an Open Access article distributed under the terms of the Creative Commons Attribution License 2.0, which permits unrestricted use, distribution, and reproduction in any medium, provided the original work is properly cited. 

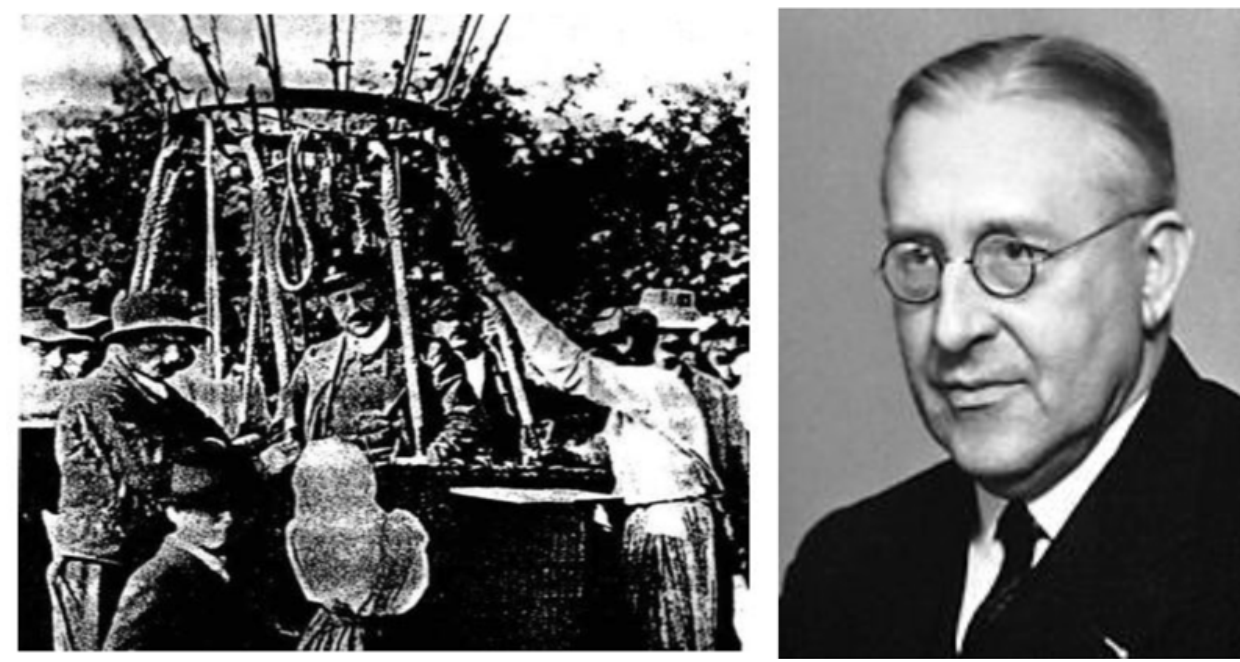

Figure 1. Victor Hess and his balloon.
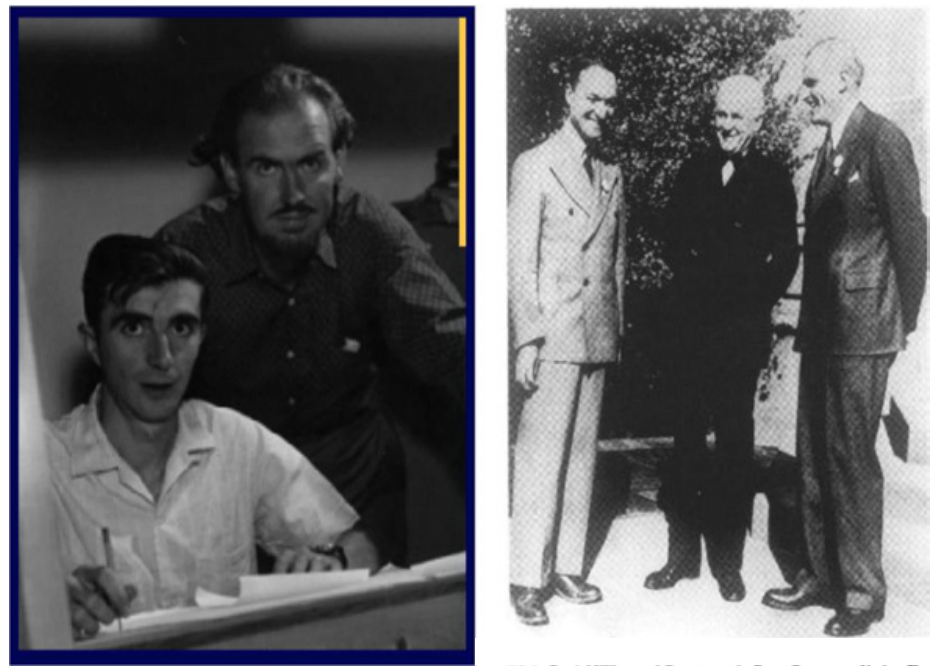

Figure 2. Left: Livio Scarsi and John Linsley. Right: Bruno Rossi, Millikan and Compton.

capable of reconstructing the footprint of an EAS of greater than $10^{15} \mathrm{eV}$ energy. This led to the Volcano Ranch array in New Mexico, whose construction was led by J. Linsley and L. Scarsi (Fig. 2). This array, with a collecting area of near $10 \mathrm{~km}^{2}$, was the first detector to show clearly that the cosmic ray spectrum extended to energies beyond $10^{19} \mathrm{eV}$. Indeed, the experimenters claimed to have detected an event beyond $10^{20} \mathrm{eV}$ in energy, though this now seems unlikely given our current knowledge of the cosmic ray intensity and the limited area and exposure of the experiment. Linsley also laid the foundation for understanding how to reconstruct and analyze EAS and made many fundamental contributions to cosmic ray phenomenology.

The cosmic ray group at the University of Leeds built a similar sized array in the UK, but utilizing water tanks instead of plastic scintillators. This was also a $10 \mathrm{~km}^{2}$ scale array but the Cherenkov light generated by the EAS particles in water gives a different response to the electrons and muons so that 
this approach was complementary to the Volcano Ranch experiment. A. Watson became the leading proponent of results from this group. Another major contribution of the Leeds group was provided by the theoretical work of M. Hillas who, among other important contributions, introduced an energy reconstruction technique that removed much of the effect of shower development fluctuations.

A Japanese group lead by M. Nagano from the Institute for Cosmic Ray Research of the University of Tokyo constructed a $20 \mathrm{~km}^{2}$ plastic scintillator array near the town of Akeno. This array also incorporated muon detectors consisting of proportional tubes behind concrete absorbers.

Meanwhile, in the USSR a group led by G. Khristiansen from Moscow State University constructed a $20 \mathrm{~km}^{2}$ array of plastic scintillators and muon detectors called the Yakutsk detector. It was unique in its time because it also incorporated an array of atmospheric Cherenkov detectors which was used to calibrate the energy scale of the events detected by the surface array. This array is still in operation and is the longest continuously operating UHE cosmic ray eperiment extant.

Initial results from these experiments all seemed to indicate that UHE cosmic rays spectrum followed a power law of $\sim$ E-3 and showed little structure or evidence for a cutoff. The Volcano Ranch data suggested a slight flattening of the spectrum above $10^{19} \mathrm{eV}$. The Haverah Park, Akeno and Yakutsk experiments all showed stronger evidence for such a flattening, since dubbed the "ankle" in the spectrum. However, since the cosmic ray intensity near $10^{20} \mathrm{eV}$ was found to be $\sim 1 / \mathrm{km}^{2}$ century, it was clear that further progress required much larger detectors. Another major issue was the reliability of hadronic and air-shower simulations regarding the absolute energy scale and the significance of shower fluctuations due to intrinsic shower development and to detector resolution.

The first attempt at a much larger detector was the SUGAR array in Australia built by a group from the University of Sydney. This was the first $100 \mathrm{~km}^{2}$ - scale detector, but its designers focused on detection of the muon component of the EAS. The uncertainty in the energy scale resulting from this choice and instrumental difficulties made the results difficult to interpret and compare with other experiments.

\section{THE AIR FLUORESCENCE ERA}

One of the major difficulties with the surface detector technique is that the magnitude and shape of the lateral distribution of particles detected by scintillation or water Cherenkov detectors is not a direct measure of the primary particle energy. Indeed, one must rely on complex Monte Carlo codes incorporating particle physics models that extend far beyond what is measured in accelerators. A more calorimetric method based on the observation of air fluorescence was initially developed by a number of groups, including K. Greisen's at Cornell and T. Suga's at the University of Tokyo. The Cornell group built a prototype "Fly's Eye" detector but were unable to observe air showers. The Japanese group may have actually detected such showers at their Dodairo observatory. The challenge was later taken up by the Utah group led by H. Bergeson, G. Cassiday and E. Loh, who were able to convincingly demonstrate the observation of air-fluorescence in coincidence with the Volcano Ranch surface detector. This led to the construction of the Fly's Eye detector at Dugway Proving Ground, which operated for about ten years. A second fly's eye detector allowed the observation of EAS in stereo and made it possible to attempt a measurement of the cosmic ray composition based on the mean value of shower maximum (Xmax) and its fluctuation. This approach is still the most reliable approach to resolving the composition issue. The Fly's Eye detector provided results on the spectrum, composition, anisotropy of cosmic rays as well as on the p-air inelastic cross-section.

\section{THE GZK CUTOFF}

The holy grail of cosmic ray physics has always been the search for the highest energy events. The spectrum as we now know, is largely featureless over almost eleven orders of magnitude in energy and each generation of experiments detected higher and higher energy events. Is there an end to this? 

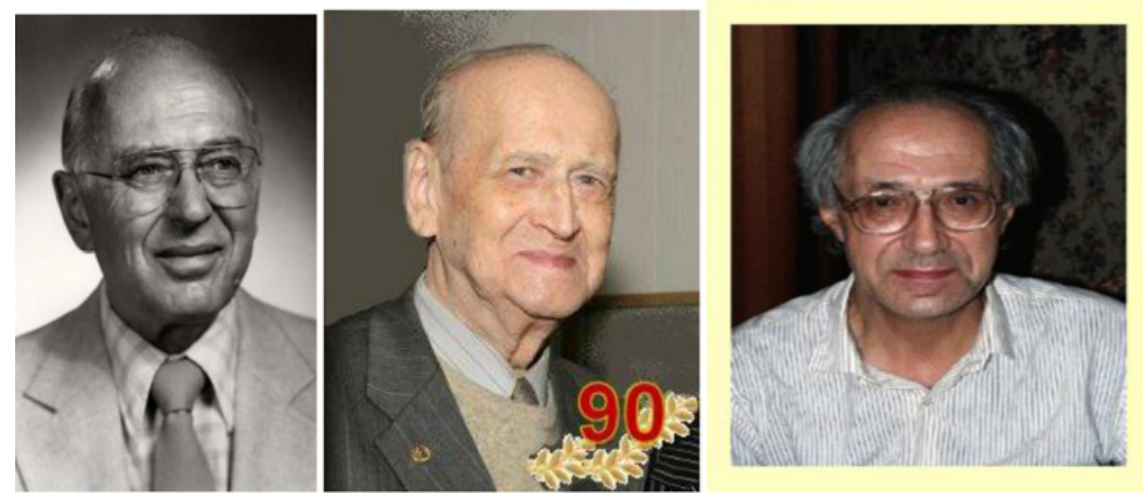

Figure 3. K. Greisen, G. Zatsepin and V. Kuzmin.

Several papers published in 1966 by K. Greisen, G. Zatsepin and V. Kuzmin (Fig. 3) clearly predicted that this would be the case. Following on the discovery of the relic microwave background radiation, it was realized that protons with energies near $10^{20} \mathrm{eV}$ and above would interact inelastically with these relic photons and lose energy as they traveled thru cosmological distances. The onset of pionphotoproduction would guarantee that a universal isotropic distribution of cosmic ray sources would exhibit a spectral cutoff at $6 \times 10^{19} \mathrm{eV}$, largely independently of the details of injection spectrum. A similar effect would occur with heavy nuclei, but in this case photo-spallation on the microwave background would be the operative mechanism. Non-observation of such a cutoff would be a serious challenge to the understanding of cosmic ray origins and propagation. None of the early surface detectors observed this cutoff, but the statistics were small and systematic issues were large. Early results from the Fly's Eye seemed consistent with a GZK cutoff, but that was about to change as well, as we will see.

\section{THE AGASA ARRAY AND THE ABSENCE OF A CUTOFF}

The AGASA array was the successor to the Akeno array as it was located in the same geographical location and was built by many of the same people. We can consider it the first modern surface array, as it used plastic scintillation counters dispersed over $100 \mathrm{~km}^{2}$ are and connected with fast fiber optics communication. The array also included muon detectors so that it had some sensitivity to composition. This experiment operated for over a decade and produced evidence of a continuation of the cosmic ray flux beyond the GZK cutoff in addition to confirming the ankle structure. A dozen or so events were detected above the GZK cutoff, with the highest energy event at $2 \times 10^{20} \mathrm{eV}$ (Fig. 4). At the same time, the final published Fly's Eye spectrum showed a single outstanding event above the cutoff at and energy of $3 \times 10^{20} \mathrm{eV}$ (Fig. 5). The AGASA data also showed unexpected clumping of events, pairs and triplets which were claimed to be statistically incompatible with fluctuations of a random background. However, these pairs and triplets as well as the post-GZK events did not point to any obvious astrophysical object of interest, such as nearby AGN's. These two results elicited a great deal of interest in the possibility of new physics at the highest energies. Everything from decays of monopolonium and cosmic strings to violation of Lorentz invariance was brought into play to explain the experimental situation.

\section{COMPOSITION RESULTS FROM THE STEREO FLY'S EYE}

John Linsley and others realized that the mean value of EAS shower maximum as well as the fluctuation of Xmax around the mean could be used to study the composition of cosmic rays. An unchanging 

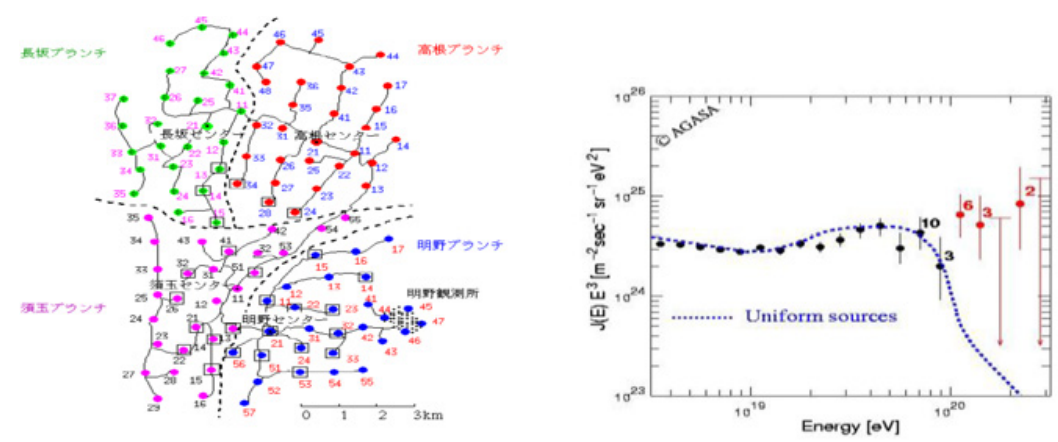

Figure 4. Left: AGASA ground array layout. Right: AGASA Spectrum and GZK expectation.
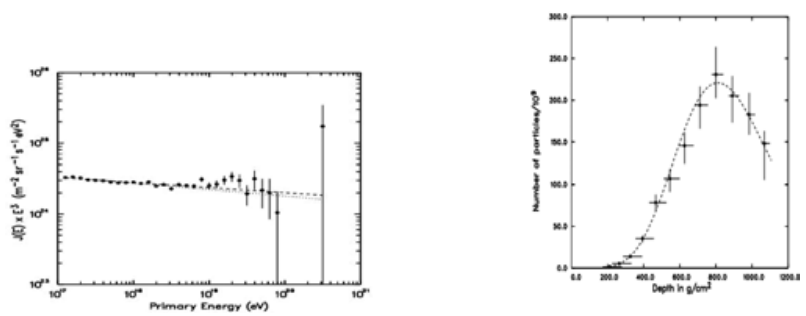

Figure 5. Left: fly's Eye monocular Spectrum. Right: highest energy Fly's Eye event.

composition would correspond to a constant "elongation rate" (slope of the mean Xmax versus $\log (\mathrm{E})$ ). Increasingly sophisticated shower development models predicted that the proton and iron elongation rates, while very similar, would be offset from each other by 70 to $100 \mathrm{gm} / \mathrm{cm}^{2}$. A transition from a light to a heavy composition, or vice versa, would effectively generate a steepening (or a hardening) of the observed elongation rate. Similarly, since the interaction of heavy nuclei can be represented as a superposition of individual nucleon showers, protons could be distinguished by their much larger fluctuations as well as deeper penetration into the atmosphere.

The first attempts at using this approach were performed by the Fly's Eye group, using a halfdecade's worth of stereo data. The resolution in shower maximum for this data set was $\sim 50 \mathrm{gm} / \mathrm{cm}^{2}$ and this value could be checked by comparing independent shower maximum measurements from the two detectors. The results pointed to a change in the cosmic ray composition from heavy to light in the energy region of the ankle in the cosmic ray spectrum (Fig. 7). There was insufficient statistics to track the composition much beyond $10^{19} \mathrm{eV}$, however.

\section{THE HIGH RESOLUTION FLY'S EYE (HIRES) EXPERIMENT}

With the successful completion of the Fly's Eye project, it was realized that substantial improvements to air-fluorescence detectors sensitivity and resolution in geometrical and shower reconstruction could be possible. The key was to reduce the angular pixel size and increase light collection efficiency. In addition, the use of modern digitizing electronics could substantially improve the signal to noise. A prototype detector was built to test these concepts. This 14 mirror prototype was sufficiently large to do physics near $10^{17} \mathrm{eV}$. In particular, advantage was taken of the close proximity (within $3 \mathrm{~km}$ ) of the CASA/MIA array (Fig. 6). This was a $1 \mathrm{~km}^{2}$ array of closely packed plastic scintillation counters and a buried array of muon detectors. While CASA/MIA was designed to do gamma-ray astronomy (searches for Cyg X-3 in particular), its muon array was large enough to provide information on the muon content 


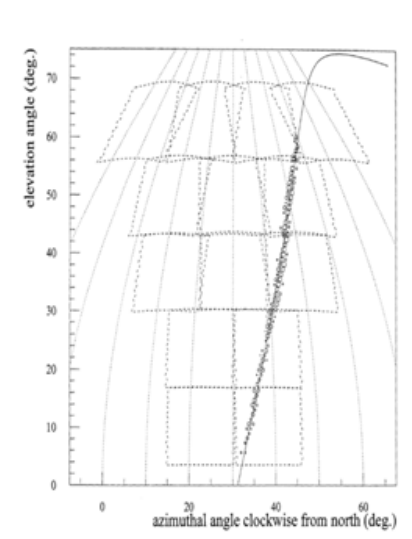

HiResl $9750.01841315 \quad$ 1995-FEB-01: 12:26:30.000 000000

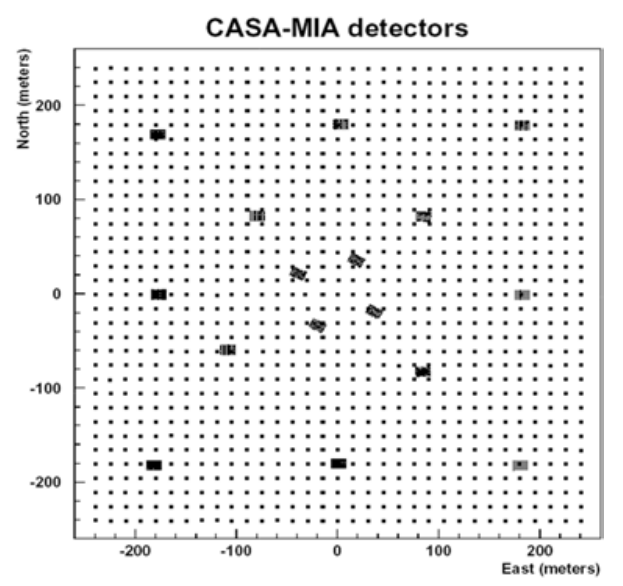

Figure 6. Left: HiRes prototype track. Right: CASA/MIA array layout. Dark rectangles are muon counters.
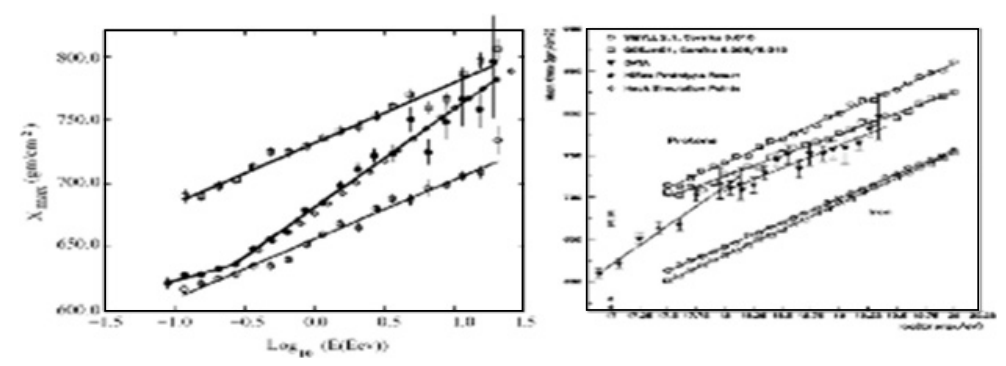

Figure 7. Left: Stereo Fly's Eye elongation rate compared to proton and Fe expectations. Right: HiRes prototype and Stereo HiRes elongation rate compared to models.

of EAS near $10^{17} \mathrm{eV}$. Events seen in coincidence by the HiRes prototype and the MIA array constituted the first significant hybrid data set. This data confirmed the conjecture that constraining the monocular (tube-timing based) air-fluorescence reconstruction with the surface detector shower front arrival time (and/or shower core position) substantially improved the reconstruction accuracy so that hybrid arrays would have very similar precision to stereo air-fluorescence detectors.

This hybrid data generally confirmed the composition trend seen in the stereo Fly's Eye experiment, but with significantly better resolution. It also hinted at an additional structure in the cosmic ray spectrum near $10^{17} \mathrm{eV}$, dubbed the second-knee. This structure also appears in the Akeno and Yakutsk surface array results (Fig. 8).

The current interpretation of the ankle structure is that it is either the effect of energy loss by protons traveling through the microwave background due to $\mathrm{e}+\mathrm{e}-$ pair production, or the transition between the termination of a galactic spectrum and a harder extragalactic component. Determining which interpretation is correct requires a good knowledge of the cosmic ray composition and energy scales. While the Fly's Eye data was consistent with the latter hypothesis, the HiRes/MIA data was more consistent with the former, since the composition change occurred at a lower energy. This difference could be easily accounted for by the lack of sufficient resolution in the early Fly's Eye data.

The full HiRes detector was conceived as a stereo detector, with two station spaced by $12.5 \mathrm{~km}$ (Fig. 9). The pixel size was 1 by 1 degree (vs. $5 \times 5$ degrees in the Fly's Eye experiment). Stereo Xmax resolution was near $30 \mathrm{gm} / \mathrm{cm}^{2}$ and the individual monocular apertures saturated at near $10,000 \mathrm{~km}^{2}$ 


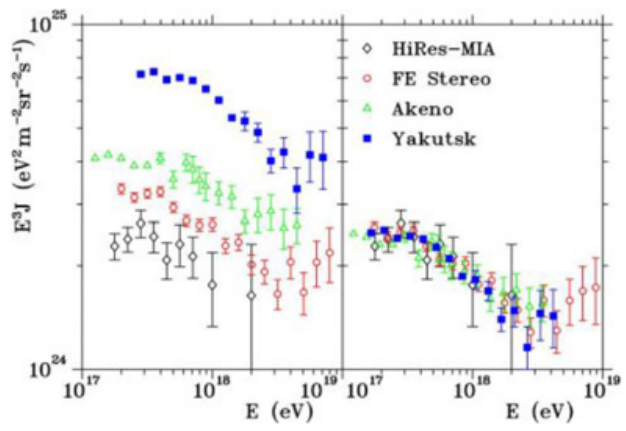

Figure 8. Evidence for a second knee. Left: spectra from four experiments. Right: spectra adjusted by changing the energy scale of each experiment so that the spectrum normalization agrees.

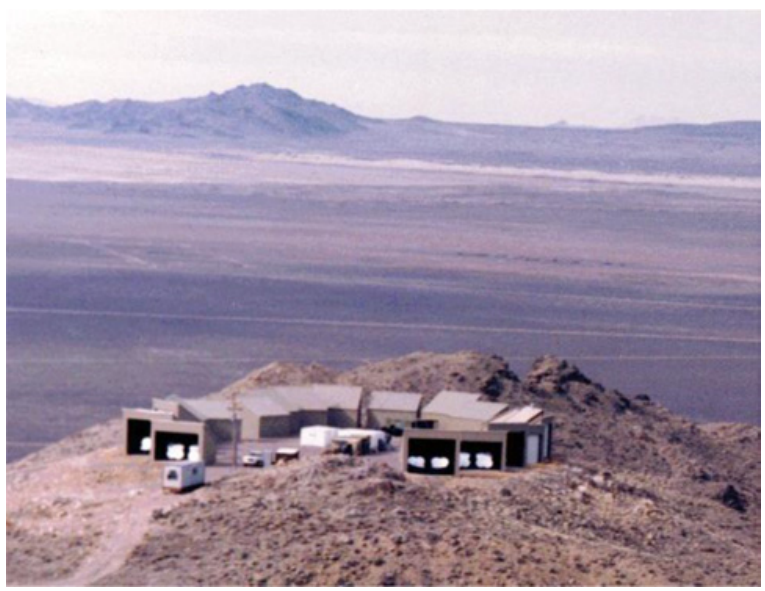

Figure 9. HiRes II detector at Dugway Proving Ground.

str. Even taking account the $10 \%$ duty cycle, this experiment had a similar sensitivity to the GZK cutoff as AGASA, but with completely different systematics.

The HiRes detectors operated for nearly a decade and the resultant spectra clearly showed the existence of a cut-off near $6 \times 10^{19} \mathrm{eV}$ (at the 5 sigma discovery level) (Fig. 10). The stereo detectors could study composition from $10^{18} \mathrm{eV}$ to $10^{20} \mathrm{eV}$, and no change in the light, mainly protonic composition was observed by either the elongation rate or fluctuation methods. The HiRes result was perfectly consistent with a protonic composition and a GZK cutoff at the predicted energy.

\section{THE PIERRE AUGER OBSERVATORY}

While the HiRes experiment was running, a much larger collaboration was building a hybrid detector in Argentina [2] (Fig. 11). This detector consists of four air-fluorescence detectors and a $\sim 3000 \mathrm{~km}^{2}$ surface array composed of water tanks. The air-fluorescence detectors have similar pixel size to HiRes but a different light collection system using Schmidt optics. The water tanks have a high efficiency for detecting muons as well as electrons via their Cherenkov light. While the surface detector is more than 30 times bigger than AGASA, the hybrid acceptance, limited to an $\sim 10 \%$ duty cycle, is a few times larger than HiRes. At energies above full efficiency for the surface detector, the hybrid acceptance 


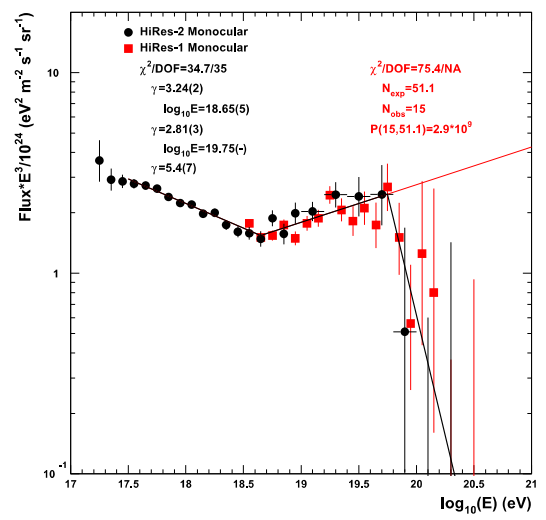

Figure 10. HiRes monocular spectra and power-law fits.

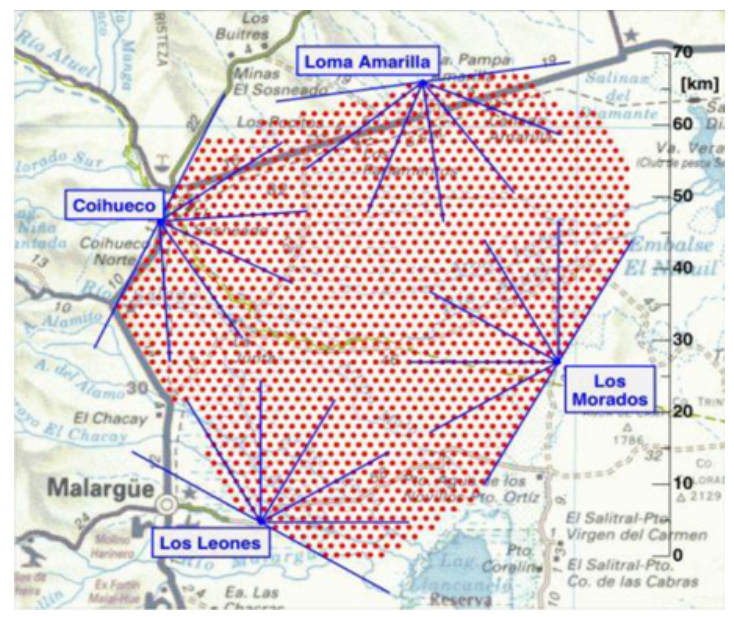

Figure 11. Layout of Pierre Auger Observatory in Argentina.

is flat in contrast to a purely air-fluorescence detector whose acceptance increases with energy. Since air-fluorescence is an inherently calorimetric technique and less dependent on complex hadronic and airshower models, the philosophy of the PAO collaboration is to use the hybrid data set to determine the energy scale of the surface detector. Once this is established, the full surface detector data set comprising the $90 \%$ of the data can be used for spectrum and anisotropy studies.

Data from this experiment will be discussed in detail in talks at this conference. The results can be summarized as confirming the observation of a GZK-like cutoff in the cosmic ray flux at the 20 sigma level, confirming a relatively light composition of cosmic rays between $10^{18}$ and $10^{19} \mathrm{eV}$ and the observation of a small but persistent correlation of arrival directions of cosmic rays with energies above $5.7 \times 10^{19} \mathrm{eV}$ with a catalogue of nearby AGN's . In contrast to HiRes, above $10^{19} \mathrm{eV}$, this experiment indicates an increasingly heavy composition, both in terms of comparison of elongation rate with models and in terms of decreasing fluctuations in Xmax. The PAO experiment observes an excess of muons relative to hadronic model expectations. This is a continuation of a trend already seen in the HiRes/MIA prototype data. 


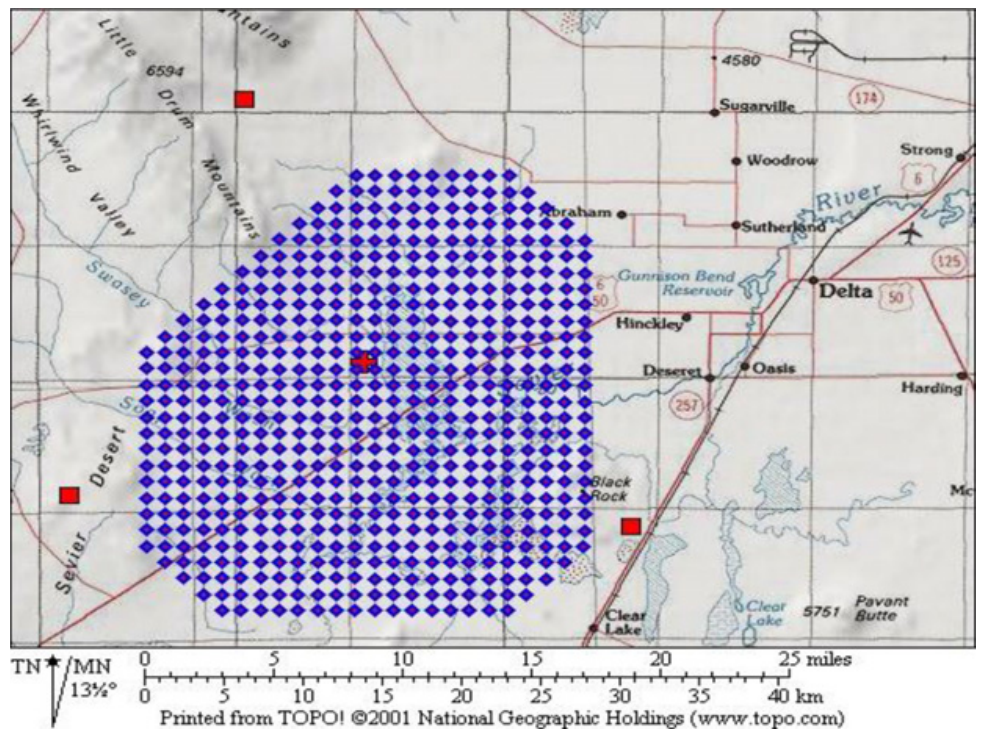

Figure 12. Layout of Telescope Array experiment in Utah.

\section{THE TELESCOPE ARRAY EXPERIMENT}

The controversy between the AGASA and HiRes results was instrumental in the formation of this new collaboration [3]. The idea was to construct an AGASA-like surface array and place it in proximity to a HiRes-like air-fluorescence detector so that the differences in the spectrum observed by HiRes and AGASA could be understood and the true behavior of the cosmic rays determined. This is essentially a hybrid experiment with a ground array $\sim 10 \times$ larger than AGASA consisting of plastic scintillators of similar area but using modern electronics. There are three air-fluorescence detector stations overlooking the surface array. Two are new designs built by the Japanese group while one is a refurbished version of the HiRes detector. This site allows the TA experiment to connect directly with the HiRes energy scale. Preliminary results presented at this meeting indicate that the surface detector with energy scaled to air-fluorescence, observes a GZK-like suppression in agreement with HiRes and PAO at the 4 sigma level. Preliminary Stereo and hybrid composition results based on the Xmax technique are completely consistent with HiRes. Anisotropy data do not show any strong correlation with nearby AGN's or the local largescale structure, but are not yet sufficiently powerful statistically to rule out such correlations.

\section{CONCLUSIONS}

The advent of the air-fluorescence technique allowed, for the first time, a convincing energy scale to be established largely independently of hadronic and air-shower models. The air-fluorescence technique also allows the determination of the depth of development of the EAS in a very direct manner thus imposing a strong constraint on composition. The cross-calibration of surface arrays using the hybrid technique makes the collection of much higher statistics data with good energy resolution possible.

More than forty years after the prediction of the GZK cutoff, an extraordinary experimental effort has culminated in the clear observation of this effect. However, the search for correlations with astrophysical objects has yet to lead to a conclusion, and the nature of cosmic rays above $10^{19} \mathrm{eV}$ remains controversial. There is much yet to be learned. This meeting is a first step in establishing a truly global effort that will be required to resolve the mystery of ultra-high energy cosmic rays. 
EPJ Web of Conferences

\section{References}

[1] References to historic primary literature can be found in the following review articles: P. Sokolsky, P. Sommers, B.R. Dawson, Phys. Rep. 217, 225 (1992); S. Yoshida and H. Dai, J. of Phys. G, 24, 905 (1998); J.W. Cronin, Rev. Mod. Phys., 71, S165 (1999); M. Nagano and A.A. Watson, Rev. Mod. Phys., 72, 689 (2000)

[2] The Pierre Auger Collab., Nucl. Inst. Meth. A523, 50 (2004); I. Allekotte et al., Nucl. Inst. Meth., A586, 409 (2008); The Pierre Auger Collab., Nucl. Inst. Meth., A620, 227 (2010)

[3] H. Tokuno et al., Nucl. Inst. Meth., 676, 54 (2012) H. Tokuno et al., Nucl. Inst. Meth., A689, 87 (2012) 